\title{
Volatilidade dos Retornos de Commodities Agropecuárias Brasileiras: um teste utilizando o modelo APARCH
}

\author{
Clailton Ataídes de Freitas ${ }^{1}$ e Thelma Sáfadi²
}

\begin{abstract}
Resumo: Este estudo analisou (2005-2013) a persistência, a alavancagem e a variância incondicional dos retornos de commodities agropecuárias ${ }^{3}$. Assim, recorreu-se ao modelo denominado APARCH. As estimativas apontaram que a alavancagem não foi confirmada nessas séries; a variância condicional foi assimétrica nos retornos do etanol, do café, do algodão, do boi gordo e do bezerro; as volatilidades mais intensas, embora com convergência às suas médias históricas, ocorreram nos retornos do açúcar, da soja, do café, do trigo, do frango e do boi gordo; as maiores volatilidades incondicionais foram dos retornos do etanol, do frango, do algodão, da soja e do açúcar.
\end{abstract}

Palavras-chaves: Efeito alavancagem; Modelo da família $\mathrm{ARCH}$; Séries agropecuárias; Potência assimétrica.

Abstract: This research analyzed (2005-2013) persistence, leverage and unconditional variance Agricultural-commodities 4 return. Therefore, we resorted to APARCH model. Estimates pointed out that leverage was not confirmed in these series; conditional variance was asymmetric in ethanol, coffee, cotton, cattle and calf's return; the most intense volatilities, although converging to its historical averages, happened to sugar, soybean, coffee, wheat, poultry and cattle; the largest unconditional volatilities were on ethanol, poultry, cotton, soybean and sugar returns.

Key-words: Leverage effect; ARCH model; Agriculture series; Asymmetric power.

http://dx.doi.org/10.1590/1234-56781806-9479005302002

1. Universidade Federal de Santa Maria (UFSM), Santa Maria, Rio Grande do Sul, Brasil. E-mail: lcv589@gmail.com.

2. Universidade Federal de Lavras (UFLA), Lavras, Minas Gerais, Brasil. E-mail: safadi@dex.ufla.br.

3. As séries estudadas foram: o açúcar, a soja, o milho, o café, o algodão, o arroz, o trigo, o frango, o boi gordo e o bezerro. A fonte dessas informações são as séries de preços do CEPEA/ESALQ, disponíveis em: http://www.cepea.esalq.usp.br. Acesso: 26/04/2013.

4. Researched series were: sugar, soybean, maize, coffee, cotton, rice, wheat, poultry, cattle and calf. The information source is the price series from CEPEA/ESALQ available at: http://www.cepea.esalq.usp.br. Access: 04/26/2013. 


\section{Introdução}

Ao reunir as vantagens comparativas naturais, o avanço tecnológico resultante de pesquisa e desenvolvimento (P\&D) em todas as etapas da produção agropecuária e política pública de apoio ao setor, o Brasil passa a ser um dos principais supridores mundiais de alimento, tornando-se competitivo na produção e exportação de vários produtos, os quais são os objetos de análise do presente estudo, a saber: açúcar, algodão, boi gordo, café, etanol, frango, milho e soja. Além desses, o País é também um grande produtor e consumidor mundial de arroz e trigo, entre outros, mas esses produtos são voltados, sobretudo, ao atendimento da demanda interna.

A Tabela 1A (Anexo 1) apresenta alguns números atuais da balança comercial do agronegócio do Brasil. Pode-se verificar que o volume de exportação no acumulado de janeiro a junho de 2013, dos produtos citados no parágrafo anterior, foi de, aproximadamente, US\$49,6 bilhões, representando, cerca de $74 \%$ de toda exportação do agronegócio do País. Nesses números, cabe ressaltar a importância da soja em grãos e do açúcar que, juntos, responderam por quase $40 \%$ dessas exportações.

Pode-se verificar também que as importações agrícolas custaram para o Brasil US\$ 8,3 bilhões e, deste total, $15 \%$ decorrem da importação de trigo e farinha de trigo. O trigo é um produto fundamental na dieta do povo brasileiro, entretanto, com base nas últimas informações disponíveis, ano safra (2014/2015), o consumo interno dessa commodity está estimado em 12,2 milhões de toneladas frente a uma produção interna de 5,9 milhões de $t^{5}$, o que torna o Brasil ainda altamente dependente das importações. Além do trigo, outro produto muito importante na culinária dos brasileiros é o arroz, cuja produção interna, na safra 2011/12, foi de cerca 12 milhões de toneladas, colocando o País na 9a posição de maior produtor mundial. Apesar desse grande volume produzido, ainda se importou, aproximadamente, US\$ 250 milhões no acumulado de janeiro a junho, correspondendo a 521 mil toneladas, ou 4,3\% da produção interna ${ }^{6}$.

Após esse rápido diagnóstico, ressaltam-se agora os principais riscos a que estão sujeitas as atividades agropecuárias. Alguns desses riscos são inerentes às atividades econômicas como flutuações de preços, contrárias às expectativas a priori do empresário; alteração das variáveis macroeconômicas, especialmente inflação, juros

5. Disponível em: <http://www.agricultura.gov.br/arq_editor/Pasta $\% 20 d e \% 20 J a n e i r o \% 20-\% 202015(1)$.pdf $>$. Acesso em: 26 fev. 2015.

6. As informações mencionadas neste parágrafo não contidas na Tabela 1 estão disponíveis em: <http://www.conab.gov. br/OlalaCMS/uploads/arquivos/13_08_09_10_43_44_boletim_portuges_agosto_2013_port.pdf $>$. Acesso: 19 ago. 2013. 
e câmbio; financeira; marketing; tecnológica; políticas econômicas específicas de apoio setorial; entre outras. Entretanto, as atividades agropecuárias enfrentam riscos adicionais em decorrência dos fatores edafoclimáticos, sazonalidade e perecibilidade dos produtos. Essa conjunção de fatores leva os agropecuaristas a sempre operarem em um ambiente de incerteza maior do que as demais atividades do sistema capitalista.

Uma das principais fontes da incerteza desses agentes é quanto ao preço futuro, uma vez que as séries de preços dos produtos agropecuários, ao longo de um determinado período, se comportam de forma cíclica. Os movimentos de baixa intensa nos preços tendem a desestimular a produção, podendo até mesmo inviabilizá-la, mesmo que se esteja operando em condições de eficiência e produtividade competitiva.

Assim, choques positivos ou negativos em um momento $t$ na série podem gerar, posteriormente, intensa volatilidade neste mercado, com ganhos ou perdas consideráveis para os produtores. Entretanto, conforme ressaltados em Ding et al. (1993), Silva et al. (2005) e Teixeira et al. (2008), os choques negativos tendem a provocar maior volatilidade na série vis-à-vis aos positivos. Esse fenômeno é conhecido na literatura como efeito alavancagem ${ }^{7}$ e decorre da característica de aversão ao risco que é, em maior ou menor grau, inerente aos agentes econômicos de modo geral. Define-se efeito alavancagem como sendo um fenômeno que surge quando períodos de quedas nos preços são seguidos por expressiva volatilidade, ao passo que, em períodos de alta nos preços, a volatilidade não é tão intensa.

Para Swaray (2002), a fonte da volatilidade é diferente quando se analisa sob a perspectiva da oferta ou da demanda. Como a demanda flutua de forma moderada de um ano a outro - em decorrência dos consumidores terem hábitos conservadores em curto período de tempo - a oferta tende a flutuar drasticamente devido, principalmente, às alterações nas variáveis climáticas, à incidência de pragas, à mudança tecnológica e às

7. Tradução do termo em inglês leverage effect. expectativas do produtor com relação ao comportamento futuro dos preços. Dessa forma, a interação da oferta de longo prazo com a demanda de curto prazo tende a resultar em acentuadas flutuações de preços.

Assim, a avaliação do comportamento dos preços das commodities, ao longo de uma janela do tempo, converte-se em uma das principais variáveis no horizonte de planejamento das atividades agropecuaristas. Em análise empírica, as séries financeiras são transformadas em taxas de retornos, o que facilita o tratamento estatístico desses processos ${ }^{8}$.

Baseando-se em Morettin e Toloi (2006), pode-se dizer que a volatilidade dos processos estocásticos é percebida de várias maneiras, como em grupos de maior ou menor variabilidade; na evolução contínua da volatilidade, mesmo com a série estacionária; na resposta assimétrica a choques positivos e negativos.

Embora a análise de volatilidade seja mais comum em ativos do mercado de capitais, nos últimos oito anos, nota-se um grande esforço dos pesquisadores no Brasil em modelar volatilidades das taxas de retornos de produtos da agropecuária brasileira. Esse esforço está materializado em vários estudos, cabendo destacar Silva et al. (2005), que examinaram o processo da volatilidade dos retornos de duas importantes commodities agrícolas, o café e a soja, por meio de modelos da classe ARCH, como GARCH, GARCH exponencial (EGARCH) e threshold ARCH (TARCH); Campos (2007), que utilizou os modelos GARCH para analisar a volatilidade das séries de retornos mensais da soja, do café, do milho e do boi gordo; Campos, Piacenti e Silva Júnior (2007), que analisaram a volatilidade das séries de retornos de produtos agroenergéticos (soja, mamona e cana-de-açúcar) por meio dos modelos ARCH e GARCH e suas extensões (TARCH e EGARCH); Teixeira et al. (2008), que aplicaram os modelos GARCH, EGARCH e TARCH para analisar os comportamentos dos retornos do cacau,

8. As vantagens dessa conversão estão apresentadas na Seção 3. 
do boi gordo, do café; Silva (2008), que analisou a volatilidade do retorno dos preços de boi gordo no estado de São Paulo (utilizando os modelo GARCH, EGARCH e TARCH); Pereira et al. (2010), que usaram uma análise comparativa dos retornos da soja, do café e do boi gordo através dos modelos EGARCH e TARCH e Value-at-Risk(VaR); de Saith e Kamitani (2012), que analisaram, por meio dos modelos ARCH, GARCH e TARCH, as volatilidades do milho, do café e da soja, considerando, também, a existência de assimetria de choques, e Moraes e Silva (2012), que examinaram a volatilidade dos retornos diários do preço à vista do café arábica por meio dos modelos EGARCH e TARCH.

Assim, não foram encontrados estudos que utilizam o modelo de potência assimétrica $\mathrm{ARCH}$ (APARCH) em simulações de séries dos retornos da agropecuária brasileira. Segundo Ding (2011), esse é o mais promissor modelo da família ARCH, pois se trata de uma generalização de outros sete modelos ${ }^{9}$ e contempla, além dos componentes ARCH e GARCH, a distribuição de cauda pesada e o efeito alavancagem. Para o autor, não existe razão óbvia de se assumir que a variância condicional seja uma função linear dos resíduos ao quadrado, como nos modelos GARCH, ou do desvio padrão condicional como no Taylor/Schwertz GARCH (TS-GARCH).

Os dados relativos aos produtos-foco da presente pesquisa têm frequência semanal e compreendem o período de junho de 2005 até abril de 2013. A escolha desse hiato temporal deve-se simplesmente a razões práticas, já que as séries apresentam tamanhos distintos. O critério adotado consistiu em escolher uma janela temporal comum a todas as séries, mas que resultasse em um número máximo de observações para que o processo de estimação não fosse comprometido com séries de tamanho restrito e também favorecesse uma análise comparativa dos resultados alcançados.

As principais contribuições da presente pesquisa se concentram em três pontos, a saber:

9. Ver Seção 2. primeiro, utiliza-se o modelo APARCH na modelagem das volatilidades dos retornos dos produtos agropecuários brasileiros, já que não foram encontrados estudos utilizando-o para tal propósito; segundo, ao invés de se trabalhar com até três produtos, como nos artigos apresentados no parágrafo anterior, estende a amostra para onze, o que permite ter uma visão mais ampla do comportamento da volatilidade dos principais commodities brutas e processadas da agropecuária brasileira e; terceiro, a consecução do estudo analisa o comportamento da volatilidade em períodos distintos dos estudos supracitados.

Assim, o presente estudo teve por objetivos estimar e analisar a variância incondicional, a persistência, o efeito alavancagem da volatilidade em cada uma das séries de retorno de produtos brutos e processados da agropecuária brasileira.

$\mathrm{O}$ presente artigo foi organizado em cinco seções, sendo a primeira constituída por esta introdução. A Seção 2 traz a fundamentação teórica do modelo utilizado. Na Seção 3 está a metodologia, onde se fez a descrição do modelo APARCH e, também a fonte e a base de dados. Na Seção 4 estão apresentados e discutidos os resultados obtidos nas estimações realizadas. Por último, na Seção 5, foram apresentadas as principais conclusões da presente pesquisa.

\section{Fundamentação teórica}

Souza (1989) define série temporal como sendo uma classe de fenômenos cujo processo observacional e consequente quantificação numérica geram uma sequência de observações distribuídas ao longo do tempo. Ao se estudar séries temporais univariadas, pode-se ter vários objetivos, conforme Moretin e Toloi (2006), como: i) avaliar a natureza e a estrutura de seu mecanismo gerador, os quais estão relacionados com o intervalo de ocorrência das observações no tempo, podendo ser contínua ou discreta; ii) realizar previsões com um ou vários passos à frente; iii) simplesmente descrever o comportamento da série, utilizando gráficos, verificando a existência 
de tendências, ciclos, variações sazonais, pontos influentes entre outros; iv) procurar a periodicidade relevantes nos dados.

Contudo, ao se estudar séries de retorno, especialmente as financeiras, é preciso estar atento ao comportamento da variância condicional. Segundo Morettin e Toloi (2006), ela evolui no tempo, não sendo adequada a utilização dos modelos lineares ARIMA na descrição desse tipo de comportamento, mas, sim, os não lineares como os da família ARCH. Para os autores, a ideia básica é que o retorno se apresenta não correlacionado serialmente, mas a volatilidade (variância condicional) depende de retornos passados por meio de uma função quadrática.

Com o surgimento dos modelos $\mathrm{ARCH}$, a volatilidade das séries passa, então, a ser estimada, tanto pela variância incondicional, que é por natureza constante, quanto pela condicional, que evolui no tempo. Neste último caso, os diversos modelos de heterocedasticidade condicional se prestam para identificá-la. Bueno (2008) afirma que a modelagem ARCH surgiu devida, principalmente, aos modelos econométricos de séries temporais, os quais consideraram apenas o primeiro momento condicional. Assim, as dependências temporais de ordens superiores, as quais expressam a presença de aglomerações de volatilidade na série, eram tratadas como perturbações aleatórias, incorporadas em seus momentos incondicionais. Já para Enders (2010), a questão central desse tipo de modelagem é que os erros não são independentes, estando correlacionados por meio do seu segundo momento.

Ainda para Enders (2010), a variância condicional segue um processo autorregressivo que resulta em erros condicionalmente heterocedásticos, isto é, se o resíduo gerado no período anterior é muito maior, ou mais próximo de zero, então, quando elevado ao quadrado, resulta em grande/pequena variância do erro, fazendo com que a variância se altere ao longo da série. Nesse sentido, a heterocedasticidade condicional presente nos resíduos torna a série de retornos um processo autorregressivo de heterocedasticidade condicional (ARCH), o que justifica a utilização dos modelos da família ARCH no diagnóstico da estrutura da volatilidade em séries temporais.

$\mathrm{O}$ modelo $\mathrm{ARCH}$ foi introduzido, inicialmente, por Engle, no início da década de 80, podendo ser matematicamente representado como segue:

$$
\begin{aligned}
& X_{t}=\sqrt{h_{t} \varepsilon_{t}} \\
& h_{t}=\alpha_{0}+\alpha_{1} X_{t-1}^{2}+\ldots+\alpha_{r} X_{t-r}^{2}
\end{aligned}
$$

em que $X_{\mathrm{t}}$ e $X_{\mathrm{t}-\mathrm{r}}$ são, respectivamente, série de retorno e série de retorno defasada, $\varepsilon_{t}$ é uma sequência de variáveis aleatórias i.i.d. com média zero e variância 1.

A partir do ARCH $(q)$ seminal proposto por Engle, surgiu uma ampla variedade de modelos com o propósito de aperfeiçoar as estimativas da estrutura da volatilidade condicional em séries temporais ${ }^{10}$. Por essa razão, o ARCH de Engel constitui a base teórica desse grande número de modelos, conhecido como modelos da família $\mathrm{ARCH}$. Um desses, o APARCH, foi utilizado no presente estudo, estando descrito na seção seguinte.

\section{Metodologia}

\subsection{A descrição do modelo}

O modelo APARCH, seguindo Ding et al. (1993) e Alencar e Sáfadi (2012), expressa cauda pesada, excesso de curtose e efeito alavancagem, e sua estrutura geral é dada por:

$$
\left\{\begin{array}{c}
\varepsilon_{t}=z_{t} \sigma_{t} \\
z_{t} \sim \mathcal{D}_{v}(0,1) \\
\sigma_{t}^{\delta}=\omega+\sum_{i=1}^{p} \alpha_{i}\left(\left|\varepsilon_{t-i}\right|-\gamma_{i} \varepsilon_{t-i}\right)^{\delta}+\sum_{j=1}^{q} \beta_{j} \sigma_{t-j}^{\delta}
\end{array}\right.
$$

em que: $\sigma_{\mathrm{t}}$ é o desvio padrão condicional da variável taxa de retorno da série em questão; $\sigma_{t}^{\delta}$ é a própria volatilidade da série, conhecida também como a variância condicional, dado o conjunto de informações disponível em $t-1 ; \omega, \delta, \alpha_{1}, \gamma_{1}$, e

10. Bollerslev (2007) apresenta um glossário dos modelos da família ARCH. 
$\beta_{1}$ são parâmetros a serem estimados; $\omega$ é o intercepto do modelo, o qual retrata o nível médio da volatilidade da variância condicional, sendo, portanto, a variância incondicional; $\delta$ representa uma transformação Box-Cox do $\sigma_{t}$, a qual possibilita estimar outras potências para o desvio padrão condicional, ao invés de apenas a potência quadrática como no GARCH, ou linear como no TS-GARCH; $\gamma_{i}$ capta efeito alavancagem, isto é, refere-se à tendência dos choques positivos e negativos terem impactos diferentes na volatilidade da série. De outra forma, $\gamma_{i}$ permite captar a resposta assimétrica da volatilidade aos retornos positivos e negativos. Assim, se o coeficiente $\gamma$ for estatisticamente significativo e positivo (resposta negativa), há evidência de efeito alavancagem, ou seja, os choques negativos ocorridos no passado têm impacto mais profundo sobre a volatilidade condicional na data corrente do que os choques positivos ocorridos no passado; se $\gamma=0$, não há nem efeito alavancagem, isto é, ambos os choques repercutirão com a mesma intensidade na volatilidade da série; $\alpha_{i}$ e $\beta_{i}$ são os coeficientes que refletem a persistência da volatilidade, sendo $\alpha_{\mathrm{i}}$ o coeficiente de reação, o qual indica o tempo necessário para que um choque na volatilidade possa ser percebido na série em questão e $\beta_{\mathrm{j}}$ mede o quanto da volatilidade do período anterior persiste no período corrente. A soma desses dois coeficientes resulta na persistência da volatilidade; $\mathcal{D}_{v}$ capta, se existir, a distribuição com cauda pesada e indica a distribuição dos erros com média 0 e variância 1 , o subíndice $v$ é o parâmetro que define a distribuição, isto é, se a distribuição $\mathcal{D}_{v}$ for uma $t$-Student simétrica, $v$ corresponde aos graus de liberdade, se for uma $t$-Student assimétrica, $v$ corresponde aos graus de liberdade e coeficiente de assimetria, mas se $\mathcal{D}_{v}$ for uma normal, $v$ será 0 .

$\mathrm{O}$ modelo APARCH pressupõe as seguintes condições: $\omega>0,0<\Sigma \alpha_{\mathrm{i}}<1,0<\Sigma \beta_{\mathrm{i}}<1$ e $\Sigma \alpha_{\mathrm{i}}+$ $\Sigma \beta_{\mathrm{i}}<1$. Cabe ressaltar, de acordo com Lamounier (2001), que as restrições se fazem necessárias para garantir que a variância condicional seja positiva e fracamente estacionária, pois argumenta o autor que, se $\Sigma \alpha_{i}+\Sigma \beta_{i} \geq 1$, os choques na volati- lidade perdurarão por um período muito longo na série, o que implica dizer que a variância condicional dos resíduos terá raiz unitária e permanecerá elevada, não apresentando reversão à sua média histórica.

Conforme Ding et al. (1993), o APARCH é uma generalização de outros sete modelos da família $\mathrm{ARCH}$, como seguem:

1. Se $\delta=2, \beta_{\mathrm{j}}=0$ e $\gamma_{\mathrm{j}}=0$, tem-se um modelo ARCH clássico;

2. Se $\delta=2$ e $\gamma_{j}=0$, tem-se um GARCH clássico de Bollerslev;

3. Se $\delta=2$ e $0 \leq \gamma_{j} \leq 1$, tem-se um modelo Glosten-Jagannathan-Runkle GARCH (GJR-GARCH);

4. Se $\delta=1$ e $\gamma_{j}=0$, tem-se um Taylor/ Schwertz's GARCH (TS-GARCH);

5. Se $\delta=1$ e $\beta_{\mathrm{j}}=0$, tem-se um modelo TARCH;

6. Se $\beta_{\mathrm{j}}=0$ e $\gamma_{\mathrm{j}}=0$, tem-se um modelo $\mathrm{ARCH}$ não linear $(\mathrm{NARCH})$;

7. Se $\delta \rightarrow 0$, tem-se um LogARCH. Algumas vezes este modelo é apresentado como Multiplicative GARCH, ou MGARCH.

\subsection{Procedimentos econométricos}

Antes de se iniciar as simulações com a modelagem ARMA, faz-se necessário testar se os processos estocásticos são estacionários. Na literatura sobre séries temporais, aparecem vários testes de raiz unitária, entre os quais se destacam os testes de Dickey-Fuller Aumentado (ADF) e o PhillipsPerron (PP). Contudo, ambos os testes têm baixa potência, tendendo a aceitar a hipótese de não estacionariedade com mais frequência, mesmo quando essa é falsa. Dessa forma, com o intuito de dar mais robustez aos resultados, realiza-se também o teste clássico ADF e os testes de Kwiatkowski, Phillips, Schmidt e Shin (KPSS) e de DickeyFuller-GLS (DFA-GLS). ${ }^{11}$

Para proceder aos testes de estacionariedade, faz-se necessário selecionar o número ótimo

11. Uma análise detalhada desses testes pode ser encontrada em Bueno (2008). 
de defasagens $(p)$ das séries. Assim, adota-se o seguinte procedimento: primeiro, determina-se um número máximo de defasagens $(p=24)$; segundo, selecionam-se as defasagens estatisticamente significativas e escolhe-se a defasagem ótima, $\left(p^{\circ}\right)$ recorrendo aos critérios Akaike (AIC) e Schwarz (BIC).

Na sequência, determina-se a ordem $p, q$ do modelo ARMA para a eliminação da correlação serial, utilizando, para tanto, o critério de AIC e BIC. Se a ordem escolhida do ARMA conseguir gerar resíduo ruído branco é porque a correlação é eliminada do processo estocástico em questão. Para o diagnóstico de ruído branco do resíduo, utiliza-se o teste de $Q(K)$ de Ljung-Box, ou teste do $\chi^{2}$. Em seguida, os resíduos são elevados ao quadrado para testar a existência do efeito $\mathrm{ARCH}$, o qual é realizado, recorrendo tanto ao teste de $\mathrm{Q}(K)$ de Ljung-Box quanto ao teste do multiplicador de Lagrange (LM) de Engle. Após a constatação da presença de heterocedasticidade na série, se for o caso, passa-se para a seleção da ordem $p$, $q$ do modelo APARCH, utilizando, novamente, os critérios de AIC e BIC.

Cabe ressaltar que os testes mencionados bem como o ajuste dos modelos APARCH ( $p$, $q)$ das séries estudadas é realizado através do software livre Gretl versão 1.9.12cvs, estando disponível em: http://gretl.sourceforge.net/win32/ index_pt.html.

\subsection{Fonte e base de dados}

Todas as informações referentes aos produtos agropecuários da presente pesquisa foram coletadas junto ao Centro de Estudos Avançados em Economia Aplicada (CEPEA) da ESALQ/USP12. As séries têm frequências semanais, com o início em 30/06/2005 e término em 26/04/2013, resultando em 409 taxas de retornos semanais para cada um dos 11 produtos analisados, a saber: etanol (R $\$$ /litro); açúcar ( $\mathrm{R} \$$ saca de $50 \mathrm{~kg}$.); soja (R\$/saca de 60kg.); milho (R\$/saca de 60kg.); café

12. Essas informações estão disponíveis em: <http://www. cepea.esalq.usp.br/>. Acesso em: 26 abr. 2013.
(R\$/saca de 60kg.); algodão (R\$/libra-peso $\left.{ }^{13}\right)$; arroz ( $\mathrm{R} \$ /$ saca de $50 \mathrm{~kg}$.), trigo (R\$/ton.), frango ( $\mathrm{R} \$ / \mathrm{kg}$.), boi gordo (R\$/arroba) e bezerro ( $\mathrm{R} \$ /$ unidade de bezerro. Dessa forma, a massa total de dados ficou constituída por 4.499 observações.

Para a maior parte dessas observações, as médias semanais foram calculadas considerando cinco dias úteis. Entretanto, devido aos feriados ou a dados faltantes em certos dias da semana, algumas médias foram calculadas com base em três ou quatro dias, e tal procedimento foi adotado para todos os 11 produtos.

Essas séries foram transformadas em taxas de retornos por meio de $y_{\mathrm{t}}=100 \ln \left(P_{\mathrm{t}} / P_{\mathrm{t}-1}\right)$, em que $P_{\mathrm{t}}$ e $P_{\mathrm{t}-1}$ são, respectivamente, os preços da commodity em questão na data $t$ e na data anterior $t-1$. A vantagem de se trabalhar com série de retorno é que essa possui propriedades estatísticas mais tratáveis (estacionariedade e ergodicidade) do que a série absoluta de preços, pois ela raramente exibe tendência e/ou sazonalidades, conforme discutido em Morettin e Toloi (2006).

\section{Resultados e discussão}

A Tabela 1 apresenta as estatísticas descritivas básicas das séries de retornos analisadas. Conforme se pode visualizar, nove produtos tiveram retornos médios variando entre $0,05 \%$ e $0,08 \%$,sendo que o maior foi proporcionado pelo bezerro, com $0,084 \%$ e o menor pelo café, com $0,013 \%$. As séries dos retornos do etanol e do frango, com desvios-padrão incondicionais respectivos de 1,69 e 1,43, foram as mais voláteis. Já as menores volatilidades incondicionais ficaram por conta do bezerro e do trigo.

A relação entre retorno e o risco, medida pelo desvio padrão, é bem estudada em ativos do mercado de capitais, sendo explicada pelo princípio de que os retornos potenciais dos investimentos estão diretamente relacionados ao risco. Assim, quando o investidor não deseja correr muito risco, obtém retornos modestos, caracteri-

13. Uma libra-peso equivale a $0,453597 \mathrm{~kg}$. 
Tabela 1. Síntese das estatísticas dos retornos $(r)$ das séries agropecuárias - período de 30/06/2005 a 26/04/2013

\begin{tabular}{cccccc}
\hline Retorno & Média & $\begin{array}{c}\text { Desvio padrão } \\
\text { incondicional }\end{array}$ & Assimetria & Curtose & $\begin{array}{c}\text { Valor p do teste de } \\
\text { normalidade } \\
\text { Shapiro-Wilk }\end{array}$ \\
\hline Etanol & 0,076 & 1,688 & $-0,712$ & 5,557 & $9,34 \mathrm{e}-016$ \\
Açúcar & 0,054 & 1,111 & $-0,261$ & 5,121 & $6,14 \mathrm{e}-015$ \\
Soja & 0,066 & 1,005 & $-0,385$ & 0,811 & 0,00058 \\
Milho & 0,027 & 1,100 & 0,701 & 1,762 & $9,94 \mathrm{e}-008$ \\
Café & 0,013 & 1,043 & $-0,085$ & 0,716 & 0,005705 \\
Algodão & 0,053 & 1,209 & $-0,575$ & 8,488 & $7,50 \mathrm{e}-018$ \\
Arroz & 0,051 & 0,901 & 1,003 & 3,150 & $1,10 \mathrm{e}-010$ \\
Trigo & 0,062 & 0,697 & 0,634 & 4,428 & $6,93 \mathrm{e}-013$ \\
Frango & 0,057 & 1,432 & $-0,504$ & 24,365 & $2,54 \mathrm{e}-021$ \\
Boi gordo & 0,070 & 0,725 & 0,296 & 2,231 & $1,21 \mathrm{e}-007$ \\
Bezerro & 0,084 & 0,619 & 0,151 & 1,251 & $1,41 \mathrm{e}-007$ \\
\hline
\end{tabular}

Fonte: Resultados da pesquisa.

zando-se como um investidor mais cauteloso ou, em uma linguagem mais técnica, avesso a risco, mas, se desejar maiores retornos, como é típico do investidor risklover, deverá naturalmente correr mais riscos. Entretanto, mais riscos não significam que os retornos desejados venham a se realizar concretamente, mas, sim, que riscos elevados potencializam mais ganhos ou perdas ${ }^{14}$. Olhando as estatísticas do desvio padrão e da taxa de retorno, Tabela 1, essa relação não se torna tão evidente, pois o produto com maior retorno, o bezerro, foi o que apresentou o menor risco, e o de maior risco, o frango, não apresentou maior retorno. De um modo geral, não se percebeu relação direta entre risco e retorno para o caso dessas 11 commodities no período estudado.

Evidentemente, outros elementos são fundamentais na tomada de decisão do produtor em continuar ou mudar de atividade, que não apenas a relação risco-retorno, como a especificidade dos ativos; o conhecimento acumulado ao longo dos anos pelos agentes com o manuseio da atividade em questão; aspectos culturais, entre outros. Aprofundar nessas outras vertentes do processo decisório do investimento foge do escopo do presente estudo.

14. Uma leitura introdutória sobre o tema está disponível em: http://www.globalinvestmentcenter.org/ index.php/investimentos-financeiros?id=56. Acesso em: 01/07/2013.
No entanto, pressupondo que os preços das commodities são formados em uma estrutura de mercado concorrencial, é esperado que as taxas de retornos dessas estejam relativamente próximas uma das outras. De modo geral, isso pode ser constatado, uma vez que todas estão próximas de zero, em até três casas depois da vírgula. Além disso, das onze séries estudadas, somente o arroz e o trigo apresentaram rentabilidades médias aquém das demais no período estudado, justamente as commodities em que o Brasil não conseguiu atingir a sua autossuficiência.

A Tabela 1 apresenta também estatísticas de curtose e assimetria, as quais são parâmetros utilizados para caracterizar uma determinada distribuição como normal, mas, para isso, o valor da curtose $(k)$ deve ser três e o da assimetria (AS) igual a zero. Conforme se pode verificar nessa tabela, as séries dos retornos do etanol, do açúcar, da soja, do algodão, do trigo e do frango são leptocúrtica $k>3$. As demais séries são platicúrticas, exceto a do arroz, com $k=3,15$, aproximando-se de uma distribuição mesocúrtica. Observando as estatísticas de assimetria, pôde-se verificar que apenas os retornos do café, com $A S=-0,085$ têm distribuição mais simétrica, e as demais são assimétricas, mas o retorno do arroz com $A S>1$ possui forte assimetria. Ao combinar os coeficientes de assimetria e curtose, é possível obter um teste 
mais formal para diagnóstico da normalidade das distribuições. A última coluna da Tabela 1 traz os resultados do teste de normalidade de ShapiroWilk. Olhando o valor $p$ da estatística desse teste, pôde-se rejeitar a hipótese $H_{0}$ de distribuição normal para o retorno dessas séries.

Antes de se efetivar a modelagem BoxJenkins, é necessário verificar formalmente se as séries em questão são estacionárias. Entretanto, como se está trabalhando com séries de retorno, espera-se, a priori, que todas elas sejam estacionárias. Assim, os testes KPSS, ADF e ADF-GLS, apresentados na Tabela 2, serviram apenas para confirmar essas expectativas prévias.

Conforme se pode vislumbrar na Tabela 2, os testes apontam que em nenhuma série há presença de raiz unitária, já que os valores calculados das estatísticas dos testes de DFA e DFA-GLS são maiores do que os seus respectivos valores críticos, levando à rejeição da hipótese nula da presença de raiz unitária no processo em questão. Da mesma forma, as estatísticas calculadas do KPSS são menores do que as estatísticas críticas, levando a não rejeição da hipótese nula de ausência de raiz unitária, corroborando, assim, com os demais testes. Portanto, os testes indicaram que todas as séries são estacionárias, estando em consonância com o esperado previamente. Na última coluna da tabela está a ordem ótima das defasa- gens usadas na realização desses testes para cada uma das séries.

Na sequência, fez-se necessário verificar se havia nas séries o problema de autocorrelação, conforme salientado por Taylor (2008). Para esse diagnóstico, foram geradas a função de autocorrelação (FAC) e a função de autocorrelação parcial (FACP) com 100 lags para cada uma das séries de retorno $(r)$. No entanto, com o intuito de facilitar a visualização dessa correlação, são apresentados, na Tabela 2A (Anexo 2), somente os resultados dos seis primeiros lags e algumas outras escolhidas aleatoriamente. As autocorrelações significativas estatisticamente, em até 5\% de significância, estão destacadas com negrito. Pode-se verificar que a primeira defasagem de autocorrelação de $r$ de todas as séries, exceto para o caso da série do bezerro, são significativas. Mesmo nesse caso, há outras defasagens de autocorrelação significativas. Assim, as séries não são estatisticamente processos iid.

Com o diagnóstico da ausência de raiz unitária, passou-se então para a eliminação da correlação serial, utilizando a modelagem Box-Jenkis. A Tabela 3 apresenta o modelo ARMA $(p, q)$, escolhido através dos critérios de AIC e BIC, para cada uma das séries estudadas, bem como as estatísticas Q $(K)$ de Ljung-Box para testar a hipótese de ruído branco dos resíduos gerado pelo ARMA

Tabela 2. Testes de raiz unitária KPSS, ADF e ADF-GLS das séries de retorno das commodities brasileiras

\begin{tabular}{ccccc}
\hline \multirow{2}{*}{ Retorno } & \multicolumn{3}{c}{ Estatística do teste } & \multirow{2}{*}{ Ordem ótima de $p^{0}$} \\
\cline { 2 - 4 } & KPSS & ADF** & ADF-GLS*** & 1 \\
Etanol & 0,0354 & $-12,549$ & $-4,2414$ & 2 \\
Açúcar & 0,1778 & $-8,1333$ & $-5,1963$ & 1 \\
Soja & 0,0930 & $-11,4024$ & $-4,2737$ & 2 \\
Milho & 0,0684 & $-10,0781$ & $-9,7868$ & 1 \\
Café & 0,1746 & $-10,9984$ & $-8,5816$ & 4 \\
Algodão & 0,0770 & $-8,3934$ & $-8,3635$ & 2 \\
Arroz & 0,0669 & $-9,0198$ & $-6,0614$ & 1 \\
Trigo & 0,1798 & $-8,9269$ & $-6,3318$ & 1 \\
Frango & 0,0299 & $-12,3005$ & $-12,3151$ & 2 \\
Boi gordo & 0,0703 & $-10,5061$ & $-9,4682$ & 4 \\
Bezerro & 0,1884 & $-6,1297$ & $-4,5286$ & \\
\hline
\end{tabular}

${ }^{*}$ Valor crítico a $5 \%=0,462 ; * *$ Valor crítico a $5 \%=-2,87 ; * *$ Valor crítico a $5 \%$ é $=-1,944$.

Fonte: Resultados da pesquisa. 
Tabela 3. Modelo $\operatorname{ARMA}(p, q)$, estatística $Q(K)$ e o Teste LM de Engle

\begin{tabular}{|c|c|c|c|c|c|}
\hline Série & Modelo & $\begin{array}{l}\mathrm{Q}(15) \\
\text { Res. }\end{array}$ & $\begin{array}{l}\mathrm{Q}(15) \\
\text { Res. quadrado* }\end{array}$ & $\begin{array}{c}\text { LM: } \chi_{\mathrm{p}}^{2}- \\
\text { Res. quadrado } p=12\end{array}$ & $\begin{array}{c}\text { Lags } \\
\text { Significativos }\end{array}$ \\
\hline Etanol & $\operatorname{ARMA}(0,1)$ & $19,0717(0,211)$ & $\begin{array}{c}81,3271 \\
(0,000) \\
\end{array}$ & $\begin{array}{c}65,052 \\
(2,667 \mathrm{e}-009) \\
\end{array}$ & MA: 1 \\
\hline Açúcar & $\operatorname{ARMA}(1,1)$ & $15.2164(0,436)$ & $\begin{array}{c}86.1449 \\
(0,000) \\
\end{array}$ & $\begin{array}{c}54,183 \\
(2,5346 \mathrm{e}-007) \\
\end{array}$ & AR:1; MA:1 \\
\hline Soja & $\operatorname{ARMA}(1,1)$ & $18,1359(0,256)$ & $\begin{array}{l}48,7870 \\
(0,000)\end{array}$ & $\begin{array}{c}36,127 \\
(0,00031)\end{array}$ & AR:1; MA:1 \\
\hline Milho & ARMA $(3,1)$ & $9.6950(0,838)$ & $\begin{array}{l}8.6611 \\
(0,895) \\
\end{array}$ & $\begin{array}{c}7,735 \\
(0,8055) \\
\end{array}$ & AR: 2,$3 ; \mathrm{MA}: 1$ \\
\hline Café & $\operatorname{ARMA}(2,2)$ & $9,7582(0,835)$ & $\begin{array}{c}64,8013 \\
(0,000)\end{array}$ & $\begin{array}{c}22,141 \\
(0,0360)\end{array}$ & AR:1 e 2; MA:1 e 2 \\
\hline Algodão & ARMA $(4,10)$ & $13,0165(0,601)$ & $\begin{array}{l}147,365 \\
(0,000)\end{array}$ & $\begin{array}{c}72,071 \\
(1,310 \mathrm{e}-010)\end{array}$ & $\begin{array}{c}\text { AR:1, 2, } 3 \text { e } 4 ; \text { MA:7, } \\
9 \text { e } 10\end{array}$ \\
\hline Arroz & ARMA $(2,7)$ & $11,0996(0,746)$ & $\begin{array}{l}97,7529 \\
(0,000)\end{array}$ & $\begin{array}{c}66,389 \\
(1,5076 \mathrm{e}-009)\end{array}$ & AR:1, 2; MA: 7 \\
\hline Trigo & $\operatorname{ARMA}(1,1)$ & $12,3305(0,654)$ & $\begin{array}{c}29,5722 \\
(0,014) \\
\end{array}$ & $\begin{array}{c}22,207 \\
(0,0353) \\
\end{array}$ & AR:1; MA:1 \\
\hline Frango & $\operatorname{ARMA}(1,1)$ & $14,8485(0,462)$ & $\begin{array}{c}91,1465 \\
(0,000)\end{array}$ & $\begin{array}{c}100,996 \\
(3,552 \mathrm{e}-016)\end{array}$ & AR:1; MA:1 \\
\hline Boi gordo & $\operatorname{ARMA}(2,0)$ & $10,0719(0,815)$ & $\begin{array}{l}94,4857 \\
(0,000)\end{array}$ & $\begin{array}{c}59,253 \\
(3,088 \mathrm{e}-008)\end{array}$ & AR:1, 2; \\
\hline Bezerro & $\operatorname{ARMA}(3,3)$ & $13,8774(0,535)$ & $\begin{array}{l}144,621 \\
(0,000)\end{array}$ & $\begin{array}{c}52,276 \\
(5,539 \mathrm{e}-007)\end{array}$ & $\begin{array}{c}\text { AR:1,2 e } 3 ; \text { MA: } 1, \\
2 \text { e } 3\end{array}$ \\
\hline
\end{tabular}

* Os valores entre parênteses indicam o valor $p$.

Fonte: Resultados da pesquisa.

$(p, q)$ selecionado. De acordo com Morettin e Toloi (2006), para a realização deste teste, em geral, basta utilizar as dez ou 15 primeiras autocorrelações. Como todas as séries estudadas são assintóticas, optou-se por utilizar Q(15), ou as primeiras 15 autocorrelações. Entretanto, autocorrelações de maior ordem também não se apresentaram estatisticamente significativas a 95\% de confiança.

Na segunda coluna da Tabela 3, estão os modelos ARMA selecionados. Evitando ser repetitivo, toma-se, por exemplo, a série do arroz. Os critérios de AIC e BIC indicaram o modelo ARMA $(2,7)$ degenerado para essa série, tendo a parte AR completa com ordem 2 e MA degenerado com somente o coeficiente da $7^{\mathrm{a}}$ defasagem significativo. Na última coluna dessa tabela, estão as defasagens estatisticamente significativas para todas as séries estudadas. Após definido o melhor modelo ARMA para cada uma das séries, são gerados os vetores dos resíduos e dos resíduos ao quadrado das mesmas, os quais são utilizados, respectivamente, para a estimação do modelo APACH $(p, q)$ e para testar o efeito ARCH.

Conforme se percebe na, Tabela 3, em todas as séries, as estatísticas $Q(K)$ para os resíduos do $\operatorname{ARMA}(p, q)$ têm valores $p$ superiores a 0,20 , estatisticamente sustentando que o modelo ARMA selecionado conseguiu eliminar a correlação serial, gerando, dessa forma, resíduo ruído branco. Frente a esse diagnóstico, o passo seguinte foi descobrir se tais vetores de resíduos apresentam heterocedasticidade. Para esse diagnóstico, recorreu-se à estatística $\mathrm{Q}(K)$ e ao teste LM de Engle nas séries dos resíduos ao quadrado. De acordo com a Tabela 3, as estimativas obtidas tanto pelo teste $\mathrm{Q}(K)$, quanto pelo LM $\operatorname{com} p=12$ lags - apontaram que, em todas as séries, exceto para os retornos do milho, com $95 \%$ de confiança, há indícios de previsibilidade, levando à rejeição da hipótese nula de homocedasticidade da vari- 
Tabela 4. Estimativas do modelo APARCH para as séries da agropecuária brasileira - 2005-2013

\begin{tabular}{|c|c|c|c|c|c|c|c|}
\hline \multirow{2}{*}{ Modelo } & \multicolumn{7}{|c|}{ Coeficiente da variância condicional $\operatorname{ARMA}(p, q)-\operatorname{APARCH}(p, q)$} \\
\hline & $\omega$ & $\alpha$ & $\gamma$ & $\beta_{1}$ & $\beta_{2}$ & $\delta$ & $v$ \\
\hline $\begin{array}{l}\text { Etanol } \\
\text { ARMA }(0,1)-\operatorname{APARCH}(2,1)\end{array}$ & $\begin{array}{c}0,5848 \\
(0,1927) \\
\end{array}$ & $\begin{array}{c}0,7204 \\
(0,2607) \\
\end{array}$ & $\begin{array}{c}-0,1924^{(\mathrm{NS})} \\
(0,1106)\end{array}$ & $\begin{array}{c}0,5383 \\
(0,1421) \\
\end{array}$ & $\begin{array}{l}-0,1858 \\
(0,0828 \\
\end{array}$ & $\begin{array}{c}1,3068 \\
(0,4132) \\
\end{array}$ & $\begin{array}{c}2,9181 \\
(1,55 \mathrm{e}-08)\end{array}$ \\
\hline $\begin{array}{l}\text { Açúcar } \\
\text { ARMA(1,1)-APARCH(1,1) }\end{array}$ & $\begin{array}{c}0,0848 \\
(0,0409) \\
\end{array}$ & $\begin{array}{c}0,3358 \\
(0,0982) \\
\end{array}$ & $\begin{array}{c}0,1350^{(\mathrm{NS})} \\
(0,1316)\end{array}$ & $\begin{array}{c}0,5394 \\
(0,0970) \\
\end{array}$ & - & $\begin{array}{c}0,8095^{\text {(NS) }} \\
(0,5475)\end{array}$ & $\begin{array}{c}5,4716 \\
(1,4473) \\
\end{array}$ \\
\hline $\begin{array}{l}\text { Soja: } \\
\text { ARMA(1, 1)-APARCH }(1,1)\end{array}$ & $\begin{array}{c}0,2465 \\
(0,0764) \\
\end{array}$ & $\begin{array}{c}0,1368 \\
(0,0367) \\
\end{array}$ & $\begin{array}{c}, 2177^{(\mathrm{NS})} \\
(0,2004)\end{array}$ & $\begin{array}{c}0,5992 \\
(0,0970) \\
\end{array}$ & - & $\begin{array}{c}0,0447^{(\mathrm{NS})} \\
(0,1430)\end{array}$ & - \\
\hline $\begin{array}{l}\text { Café } \\
\text { ARMA(2, 2)-APARCH }(1,1)\end{array}$ & $\begin{array}{l}0,0712^{(\mathrm{NS})} \\
(0,0465)\end{array}$ & $\begin{array}{c}0,1088 \\
(0,0479) \\
\end{array}$ & $\begin{array}{c}-0,074^{(\mathrm{NS})} \\
(0,1884) \\
\end{array}$ & $\begin{array}{c}0,8276 \\
(0,0768) \\
\end{array}$ & - & $\begin{array}{c}1,7290 \\
(0,7224) \\
\end{array}$ & $\begin{array}{c}9,8650 \\
(4,6078) \\
\end{array}$ \\
\hline $\begin{array}{l}\text { Algodão } \\
\text { ARMA }(4,10)-\operatorname{APARCH}(1,1)\end{array}$ & $\begin{array}{c}0,0680^{(\mathrm{NS})} \\
(0,0397)\end{array}$ & $\begin{array}{c}0,6107 \\
(0,2584) \\
\end{array}$ & $\begin{array}{c}0,1088^{(\mathrm{NS})} \\
(0,0873)\end{array}$ & $\begin{array}{c}0,3750 \\
(0,1894) \\
\end{array}$ & - & $\begin{array}{c}2,7922 \\
(1,5126) \\
\end{array}$ & $\begin{array}{c}4,0753 \\
(0,8903) \\
\end{array}$ \\
\hline $\begin{array}{l}\text { Arroz } \\
\text { ARMA(2,7)-APARCH}(1,1)\end{array}$ & $\begin{array}{c}0,0416 \\
(0,0157) \\
\end{array}$ & $\begin{array}{c}0,2551 \\
(0,0752) \\
\end{array}$ & $\begin{array}{c}-0,2055^{(\mathrm{NS})} \\
(0,1165) \\
\end{array}$ & $\begin{array}{c}0,5322 \\
(0,1421) \\
\end{array}$ & - & $\begin{array}{c}1,9621 \\
(0,7917) \\
\end{array}$ & - \\
\hline $\begin{array}{l}\text { Trigo } \\
\text { ARMA(1,1)-APARCH(1, 1) }\end{array}$ & $\begin{array}{l}0,0216^{(\mathrm{NS})} \\
(0,0146)\end{array}$ & $\begin{array}{c}0,1745 \\
(0,0721) \\
\end{array}$ & $\begin{array}{c}-0,1889^{(\mathrm{NS})} \\
(0,1668 \\
\end{array}$ & $\begin{array}{c}0,8140 \\
(0,0803)\end{array}$ & & $\begin{array}{c}0,9197 \\
(0,4646) \\
\end{array}$ & $\begin{array}{c}4,1762 \\
(0,9119) \\
\end{array}$ \\
\hline $\begin{array}{l}\text { Frango } \\
\text { ARMA }(1,1)-\operatorname{APARCH}(1,1)\end{array}$ & $\begin{array}{c}0,4408 \\
(0,1903) \\
\end{array}$ & $\begin{array}{c}0,4503 \\
(0,1275) \\
\end{array}$ & $\begin{array}{c}0,0190^{(\mathrm{NS})} \\
(0,0967)\end{array}$ & $\begin{array}{c}0,4084 \\
(0,1698) \\
\end{array}$ & - & $\begin{array}{c}0,9831 \\
(0,3460) \\
\end{array}$ & $\begin{array}{c}4,7616 \\
(1,1575) \\
\end{array}$ \\
\hline $\begin{array}{l}\text { Boi gordo } \\
\text { ARMA }(2,0)-\operatorname{APARCH}(1,1)\end{array}$ & $\begin{array}{c}0,0293 \\
(0,0131) \\
\end{array}$ & $\begin{array}{c}0,3546 \\
(0,0919) \\
\end{array}$ & $\begin{array}{c}0,0067^{(\mathrm{NS})} \\
(0,0985)\end{array}$ & $\begin{array}{c}0,6331 \\
(0,0819) \\
\end{array}$ & - & $\begin{array}{c}1,5506 \\
(0,6816) \\
\end{array}$ & $\begin{array}{c}6,5180 \\
(2,1491) \\
\end{array}$ \\
\hline $\begin{array}{l}\text { Bezerro } \\
\text { ARMA }(3,3)-\operatorname{APARCH}(1,1)\end{array}$ & $\begin{array}{c}0,0079^{\text {(NS) }} \\
(0,0048)\end{array}$ & $\begin{array}{c}0,4938 \\
(0,1293)\end{array}$ & $\begin{array}{c}0,0146^{(\mathrm{NS})} \\
(0,0801)\end{array}$ & $\begin{array}{l}0,7226 \\
(0,077)\end{array}$ & - & $\begin{array}{c}0,6474 \\
(0,0649)\end{array}$ & $\begin{array}{c}4,6066 \\
(1,1390)\end{array}$ \\
\hline
\end{tabular}

Nota: Os parâmetros não significativos estão sobrescritos com ${ }^{(\mathrm{NS})}$.

Fonte: Resultados da pesquisa.

ância condicional. Portanto, o efeito ARCH está presente nos resíduos de todas essas séries, exceto para o caso do milho, pois, com o valor da estatística $\mathrm{Q}(15)=8.6611$ e valor da probabilidade $(p=$ $0,8055)$ do LM, pode-se afirmar estatisticamente que tal vetor de resíduo tem variância condicional homocedástica, não justificando, assim, a utilização dos modelos da família ARCH.

Passou-se, a seguir à estimação do modelo $\operatorname{ARMA}(p, q)-\operatorname{APARCH}(p, q)$ para as séries heterocedástica. A Tabela 4 apresenta somente os resultados finais da especificação $\operatorname{ARMA}(p, q)$ - $\operatorname{APARCH}(p, q)$, obtidos recorrendo aos critérios de AIC e BIC, após inúmeras simulações. Cabe ressaltar, que, para a seleção do melhor modelo de uma determinada série, são simuladas, além das distribuições condicionais (normal, $t$-Student simétrica e $t$-Student assimétrica), as diferentes ordens de defasagens $\mathrm{GARCH}$ e $\mathrm{ARCH}$ do APARCH. Outro ponto que merece ser ressaltado, é a utilização do método hessiano para estimar a matriz de covariância, gerado de forma interativa no default do Gretl. O modelo escolhido é o que apresenta o menor valor dos critérios AIC e BIC, sempre tendo como referência a escolha de modelos mais parcimoniosos.

Um resultado comum a todas as séries, retratado na Tabela 4, é a não significância estatística do efeito alavancagem $\gamma$, apontando que quedas ou subas nos preços dessas commodities tendem a ter o mesmo efeito sobre a volatilidade. De imediato, esse resultado parece incoerente, visto que o efeito alavancagem em séries financeiras é fato estilizado. Infelizmente, não foram encontrados estudos utilizando o APARCH em séries dos retornos da agropecuária brasileira em outra janela temporal, o que permitiria comparar os resultados alcançados com o presente estudo. Entretanto, o efeito alavancagem é estimado em alguns estudos através da modelagem EGARCH e $\mathrm{TGARCH}^{15}$, entre os principais se destacam Silva et al. (2005), que, ao longo de 1967-2002, não diagnosticaram o efeito alavancagem nas séries

15. A estimação do efeito alavancagem difere nos modelos TGARCH e EGARCH. Assim, se esse efeito estiver presente em uma determinada série e for utilizado o TGARCH, o sinal de $\gamma$ será positivo, ao passo que, se for utilizado EGARCH, o sinal de $\gamma$ será negativo. 
café e soja. Campos et al. (2007), no período de 1967 a 2006, não encontraram o efeito alavancagem na série de retorno da soja, apesar de tê-lo encontrado nos retornos da mamona e da cana-de-açúcar. Pereira et al. (2010), para o período 1997 a 2008, encontraram resultados convergentes ao de Silva et al. (2005) para os mesmos produtos, mas não para a série do boi gordo, dado que os autores não conseguiram modelos bem ajustados capazes de eliminar a autocorrelação e/ou efeito ARCH da variância.

O presente estudo se diferencia dos supracitados, pelo menos em dois aspectos: o modelo APARCH é mais complexo vis-à-vis TGARCH e EGARCH e o período de análise é distinto, mas, mesmo assim, os resultados aqui estimados, corroboraram com os encontrados por esses estudos, isto é, a não existência do efeito alavancagem para as séries do café, da soja e do boi gordo.

Além da não significância estatística do parâmetro $\gamma$, o coeficiente da potência assimétrica $\delta$, também não se apresentou significativo nas séries do retorno do açúcar e da soja. A combinação desses dois resultados indica que especificações mais restritas do APARCH, tal como MGARCH, poderiam ser viáveis na estimação da volatilidade dessas séries. Com a potência estatisticamente próxima a um, têm-se os retornos do frango $(0,98)$, do trigo $(0,92)$, e do açúcar $(0,81)$, o que estaria apontando para uma especificação mais simples do APARCH, dependendo, logicamente, dos valores dos demais parâmetros, como o modelo TS-GARCH. Com a potência assimétrica estatisticamente próxima a dois, teve-se apenas a série do arroz, sinalizando para um ARCH clássico ou GARCH de Bollerslev. Para as demais séries, teve-se apoio estatístico para afirmar $\delta$ é diferente de um ou dois.

Os efeitos ARCH e GARCH do APARCH, captados pelos coeficientes $\alpha$ e $\beta$, respectivamente, apresentaram-se significativos em termos estatísticos em todas as séries. O parâmetro $\alpha$ capta a reação da volatilidade a um choque na série de retorno. Assim, quanto maior/menor for o valor desse coeficiente, maior/menor o tempo que irá persistir um choque sobre a volatilidade na série.
Olhando para a Tabela 4 , nota-se que um choque nas séries dos retornos do etanol $(\alpha=0,7204)$ e algodão $(\alpha=0,6107)$ terá efeito por vários períodos na volatilidade destes respectivos retornos. Os menores coeficientes de reação estão relacionados às séries dos retornos do café $(\alpha=0,1088)$, soja $(\alpha=0,1368)$ e trigo $(\alpha=0,1745)$.

A persistência ou inércia da volatilidade é diagnosticada pelo $\beta$. Conforme retratado na tabela anterior, as séries de retornos com os maiores coeficientes de persistência foram as do café $(\beta=0,8276)$, do trigo $(\beta=0,814)$, do bezerro $(\beta=$ $0,723)$ e do boi gordo $(\beta=0,633)$, e as com os menores coeficientes, relacionados ao do algodão ( $\beta=0,3750)$, do frango $(\beta=0,4084)$ e do açúcar $(\beta=0,5394)$. Para não tornar a leitura cansativa, toma-se, por exemplo, o caso dos valores extremos do café e do algodão. Nestes casos, o coeficiente de persistência indica que mais de $80 \%$ de um choque gerado na série do retorno do café no tempo $t$-1 persistirá no tempo $t$, enquanto, para a do algodão, essa persistência é de pouco mais de $35 \%$. A soma dos parâmetros $\alpha$ e $\beta$ evidencia quão volátil é uma série. A Tabela 4 revela que as séries do etanol e do bezerro não obedecem à restrição $\sum \alpha_{\mathrm{i}}+\sum \beta_{\mathrm{i}}<1$, o que retrata que choques na volatilidade permanecem na série por longo período, não apresentando, assim, reversão à sua média histórica, devido à presença de raiz unitária.

As demais séries, excetuando as do arroz e da soja, possuem alta volatilidade, já que a soma dos coeficientes de reação $(\alpha)$ e de persistência $(\beta)$ são superiores a 0,7 , revelando que, após um choque, o processo de reversão da variância condicional para seu valor médio tende a ser lento, entretanto, como não existe raiz unitária, a tendência é que isso ocorra. Em outras palavras, os efeitos de um choque nessas séries agropecuárias tendem a afetar a volatilidade desses retornos por vários períodos, mas espera-se a sua reversão para a sua média histórica. $\mathrm{O}$ baixo valor da soma de $\alpha$ e $\beta$ da série dos retornos do arroz indica que qualquer choque nesta série tende a se dissipar rapidamente, afetando a volatilidade desses retornos somente por um curto período de tempo. 
Os principais estudos disponíveis na literatura sobre a estimativa da persistência da volatilidade são para os retornos dos seguintes produtos: café, soja, boi gordo, milho, mamona e cana-de-açúcar. As estimativas obtidas, no presente estudo, para tais retornos, exceto para os casos da mamona e da cana-de-açúcar, são café $(0,9364)$; soja $(0,736)$; boi gordo $(0,9877)$; o milho não apresenta efeito $\mathrm{ARCH}$, conforme já salientado. Saith e Kamitani (2012), usando GARCH e TARCH, encontraram para o milho $(0,868)$; para o café $(0,975)$ e para a soja $(0,978)$. Pereira et al. (2010), recorrendo ao EGARCH e TARCH, ao longo de 1997 a 2008, também obtiveram intensa volatilidade para os retornos da soja $(0,860)$, do café $(0,89)$ e do boi gordo $(0,87)$. Campos $(2007)$, usando um GARCH no período de 1967 a 2006, estimou intensa volatilidade dos retornos do café $(0,962)$, do milho $(0,889)$ e do boi gordo $(1,044)$. Campos et al. (2007), recorrendo ao GARCH, EGARCH E TARCH, encontraram, no período de 1967 a 2006, intensa volatilidade nos retornos da soja acima de 0,73; Silva et al. (2005), para o período de 1967 a 2006, utilizando EGARCH e $\mathrm{TARCH}$, encontraram alta volatilidade para as séries dos retornos do café $(0,88)$ e da soja $(0,95)$.

Comparando de um modo geral os resultados dos estudos supracitados com os aqui alcançados, pode-se dizer que as séries dos retornos do café e do boi gordo apresentaram intensa volatilidade acima de 0,8 , apontando que choques sobre a volatilidade tendem a levar um longo tempo para se dissiparem. No caso dos retornos da soja, a estimativa obtidas de uma persistência de volatilidade em torno de 0,7 ficou um pouco aquém das apresentadas por Saith e Kamitani (2012) e Pereira et al. (2010), mas se pensa que tal diferença não seja expressiva. No entanto, isso parece indicar que a série de retorno da soja nos últimos anos tornou-se menos volátil.

O parâmetro $\omega$ capta o nível de volatilidade incondicional das séries dos retornos. Conforme a Tabela 4 , as séries do etanol $(\omega=0,58)$, do frango $(\omega=0,38)$ da soja $(\omega=0,29)$ e do açúcar $(\omega=0,11)$ são as que apresentaram os maiores valores de $\omega$, o que poderia apontar para volatilidades incon- dicionais mais intensas nessas séries. Quando são comparados os valores desses parâmetros com os da Tabela 1, os resultados são coerentes para o caso do etanol, do frango e do açúcar, mas, aparentemente, divergem com relação à série dos retornos do algodão, que, conforme a Tabela 1, apresentou o terceiro maior desvio padrão incondicional. Contudo, deve-se ter cautela ao interpretar esse parâmetro, pois, como $\delta=2,692$, o valor de $\omega=0,0728$ está mais próximo da variância do que do desvio padrão, fazendo, então, essa correção $\left(0,0728^{1 / 2,692}\right)$, chega-se ao valor de 0,378 , que corresponde ao $3^{\circ}$ maior desvio padrão incondicional estimado pelo modelo APARCH $(1,1)$, estando, portanto, condizentes com o resultado da Tabela 1. As demais séries apresentaram volatilidades baixas, corroborando com os resultados apresentados anteriormente na Tabela 1.

Cabe ressaltar que no processo de simulação foram utilizadas as distribuições normal, $t$-Student simétrica e $t$-Student assimétrica na estimação dos regressores da variância dos resíduos, conforme salientado anteriormente. Para a escolha da distribuição mais adequada para o modelo em questão, recorreu-se aos critérios AIC e BIC. De acordo com esses critérios, a distribuição $t$ simétrica ajustou melhor as séries do etanol, do açúcar, do café, do algodão, do trigo, do frango, do boi gordo, do bezerro, enquanto a distribuição normal foi mais indicada nos ajustes das séries da soja e do arroz. A penúltima coluna da Tabela 4 apresenta as estimativas do parâmetro $v$ para as distribuições $t$-Student, e, conforme se pode evidenciar, todos eles são significativos.

\section{Conclusões}

Buscou-se, no presente estudo, estimar e analisar a variância incondicional, a persistência e o efeito alavancagem da volatilidade para as principais séries de retornos das commodities brutas e processadas da agropecuária brasileira.

Um dos resultados obtidos com a modelagem $\operatorname{APARCH}(p, q)$ é contrário às expectativas a priori, ao não confirmarem a existência de efeito alavan- 
cagem em nenhuma das séries estudadas, fato esse estilizado em séries financeiras. Isso revelou que as oscilações (queda ou suba nos preços) têm o mesmo efeito sobre a volatilidade.

Com relação ao coeficiente da potência assimétrica $\delta$, diagnosticou-se que ele não foi significativo nas séries do retorno do açúcar e da soja, indicando que especificações mais simples do APARCH poderiam ser viáveis na estimação da volatilidade de tais séries. Ainda no sentido de se utilizar modelos mais simples para a estimação da volatilidade, notou-se que as séries dos retornos do frango, do trigo e do açúcar apresentaram o valor $\delta$ próximo a 1, e a do arroz, próximo a 2 , o que estaria apontando, também, para modelos mais simples do APARCH. Entretanto, para as séries de retorno do etanol, do café, do algodão, do boi gordo e do bezerro, tem-se apoio estatístico em afirmar que $\delta$ é diferente de 1 ou 2.

Notou-se que os retornos dos produtos agropecuários estudados, em geral, apresentaram intensa volatilidade condicional, sinalizando para a tendência de ocorrência de aglomerações de volatilidade, isto é, períodos de baixa volatilidade seguidos por períodos de oscilações mais proeminentes. Isso foi percebido em todas as séries, com exceção da do arroz. As séries dos retornos do etanol e do bezerro revelaram que, após um choque nas suas variâncias condicionais, não há reversão à sua média histórica. As séries do açúcar, da soja, do café, do trigo, do frango e do boi gordo possuem volatilidade intensa, mas, após um choque, apesar de lento, ocorre o processo de reversão da variância condicional para seu valor médio. No caso específico do retorno do arroz, os choques sobre a volatilidade tendem a se dissiparem rapidamente, afetando a variância condicional somente por um curto período de tempo.

Outro resultado obtido diz respeito às estimativas da volatilidade incondicional das séries dos retornos. Percebeu-se que as séries do etanol, do frango, do algodão, da soja e do açúcar são as que apresentaram maiores volatilidades incondicionais. As séries do café, do arroz, do trigo, do boi gordo e do bezerro apresentaram volatilidades incondicionais baixas.
Os critérios utilizados na seleção dos modelos indicaram que a distribuição $t$ simétrica ajustou melhor as séries do etanol, do açúcar, do café, do algodão, do trigo, do frango, do boi gordo e do bezerro, enquanto a distribuição normal ajustou melhor as dos retornos da soja e do arroz.

Uma questão que se coloca e que precisa ser investigada do ponto de vista estatístico é a seguinte: o modelo APARCH é, realmente, superior aos modelos EGARCH e TARCH, ou a outros modelos na estimação dos efeitos relacionados ao efeito alavancagem? Evidentemente que simulações estatísticas precisariam ser realizadas, o que fugiria do escopo do presente estudo, mas se recomenda esse exercício para futuras pesquisas.

\section{Referências}

ALENCAR, A. P. e SÁFADI, T. Volatility of main stock indexes: similarities and differences. International Journal of Statistics and Economics, v. 9, n. A12, p. 1-12, 2012.

BOLLERSLEV, T. Glossary to ARCH (GARCH), Papers, North Caroline, United States, feb.2009 p.01-41. Disponível em: < http://public.econ.duke.edu/ boller/ Papers/glossary_arch.pdf $>$. Acesso em: 20 out. 2013

BUENO, R. L. S. Econometria de séries temporais. São Paulo: Cengage Learning, 2008.

CAMPOS, K. C. Análise da volatilidade de preços de produtos agropecuários no Brasil. Revista de Economia e Agronegócio, v. 5, n. 3, p. 303-328, 2007.

CAMPOS, K. C., PIACENTI, C. A. e SILVA JUNIOR, A.G. Agroenergia: a questão da volatilidade de preços e o efeito alavancagem dos produtos agrícolas. Revista de PolíticaAgrícola, v. 16, n. 03, p. 34-48, 2007.

DING, D. Modeling of market volatility with APARCH model. Project Report n. 06/2011, Uppsala University Department of Mathematics, Sweden, Uppsaka, mai. 2011. Disponível em: <http://www.diva-portal.org/ smash/get/diva2:417608/FULL TEXT01.pdf>. Acesso: 20 ago. 2013.

DING, Z., GRANGER, C. W. J. e ENGLE, R. F. A long memory property of stock market returns and a new model.Journal of Empirical Finance, v. 1, p. 83-106, 1993.

ENDERS, W. Applied econometric time series. 3. ed. New York: John Wiley \& Sons, Inc., 2010. 
ENGLE, R. F. Autoregressive conditional heteroscedasticity with estimates of the variance of United Kingdom inflation. Econométrica, v. 50, p. 9871007, 1982.

LAMOUNIER, W. M. Comportamento dos preços no mercado "spot" de café do Brasil: análise nos domínios do tempo e da freqüência. 2001. 112 f. Tese (Doutorado em Economia Aplicada) - Programa de Pós-Graduação em Economia Aplicada, Universidade Federal de Viçosa, Viçosa - MG.

MINISTÉRIO DA AGRICULTURA, PECUÁRIA E ABASTECIMENTO - MAPA. Dados básicos de economia agrícola. Brasília, jul. 2013. 51p. Disponível em: < http:// www.agricultura.gov.br/arq_editor/Pasta $\% 20$ de $\% 20$ Julho\% 20\% 202013.pdf>. Acesso em: 14 mar. 2015.

MORAES, L. P. e SILVA, C. A. G. A volatilidade do preço do café: um estudo dos modelos heterocedásticos. In: ENCONTRO NACIONAL DE ENGENHARIA DE PRODUÇÃO. 31., 2011, Belo Horizonte. Inovação Tecnológica e Propriedade Intelectual: Desafios da Engenharia de Produção na Consolidação do Brasil no Cenário Econômico Mundial, Belo Horizonte MG: ENEGEP, 2011. Disponível em: < http://www.abepro.org.br/biblioteca/ enegep2011_TN_STO_140_887_18973.pdf >. Acesso em: 10 out. 2013.

MORETTIN, P. A. e TOLOI, C. M. C. Análise de séries temporais. 2. ed. São Paulo: EgardBlucher, 2006.

PEREIRA, V. F., LIMA, J. E., BRAGA, J. M. e MENDONÇA, T. G. Volatilidade condicional dos retornos de commodities agropecuárias brasileiras. Revista de Economia, v. 36, n. 03, p. 73-94, 2010.

SILVA, C. A. G. e MORAES, L. P. Análise da dinâmica da volatilidade dos preços à vista do café arábica: aplicação dos modelos heteroscedásticos. In: CONFERÊNCIA EM GESTÃO DE RISCO E COMERCIALIZAÇÃO DE COMMODITIES, 2., 2012, São Paulo. Conferência CGCC, São Paulo SP: Instituto Educacional BM\&FBOVESPA, 2012. Disponível em: <http://www.bmfbovespa. com.br/CGRCC/download/Analise-da-DinamicadaVolatilidade.pdf>. Acesso em: 03 jul. 2013.

SILVA, W. S., SÁFADI, T. e CASTRO JÚNIOR, L. G. de. Uma análise empírica da volatilidade do retorno de commodities agrícolas utilizando modelos ARCH: os casos do café e da soja. Rio de Janeiro: Revista de Economia e Sociologia Rural, v. 43, n. 1, p. 119-134, 2005.

SILVA, C. A. G. Análise da volatilidade dos preços de boi gordo no Estado de São Paulo: uma aplicação dos modelos GARCH. In: ANAIS DO CONGRESSO DA SOCIEDADE BRASILEIRA DE ECONOMIA E SOCIOLOGIA RURAL (SOBER). 46., 2008, Rio Branco. Anais Amazônia, mudanças globais e agronegócio: o desenvolvimento em questão, Rio Branco AC: SOBER, 2008. Disponível em: <http://www.sober.org.br/ palestra/6/429.pdf>. Acesso em: 02 ago. 2013.

TEIXEIRA, S. G. et al. Dinâmica da volatilidade do retorno das principais commodities brasileiras: uma abordagem dos modelos ARCH. In: ANAIS DO CONGRESSO DA SOCIEDADE BRASILEIRA DE ECONOMIA E SOCIOLOGIA RURAL (SOBER). 46., 2008, Rio Branco, AC (Brasil). Anais Amazônia, mudanças globais e agronegócio: o desenvolvimento em questão, Rio Branco AC: SOBER, 2008. Disponível em: < http:// www.sober.org.br/palestra/9/544.pdf $>$. Acesso em: 02 ago. 2013.

SAITH, W. e KAMITANI, T. L. Volatilidade e assimetria de choques no mercado Agropecuário brasileiro um uso dos modelos da família GARCH. Tecnologia e Ciência Agropecuária, v. 6, n. 1, p. 1-07, 2012.

SOUZA, R. C. Modelos estruturais para previsão de séries temporais: abordagens clássica e bayesiana. In: COLÓQUIO BRASILEIRO DE MATEMÁTICA. 17, Colóquio IMPA, 1989, Rio de Janeiro, RJ: IMPA, 1989.

SWARAY, R. B. Volatility of primary commodity prices: some evidence from agricultural exports in SubSaharan Africa. Discussion paper in economics, n. 6/2002. Department of Economics and Related Studies, University of York, York, England, 2002. Disponível em: <http://www.york.ac.uk/media/economics/documents/ discussion papers/2002/0206.pdf> . Acesso: 21 ago. 2013.

TAYLOR, J. S. Modeling financial time series. 2. ed. Singapore: World Scientific Printers, 2008.

VEIGA, H. Financial stylized facts and the Taylor-Effect in Stochastic Volatility Models. Economics Bulletin, v. 29, n. 1, p. 2-11, 2009. 
226 - Volatilidade dos Retornos de Commodities Agropecuárias Brasileiras: um teste utilizando o modelo APARCH

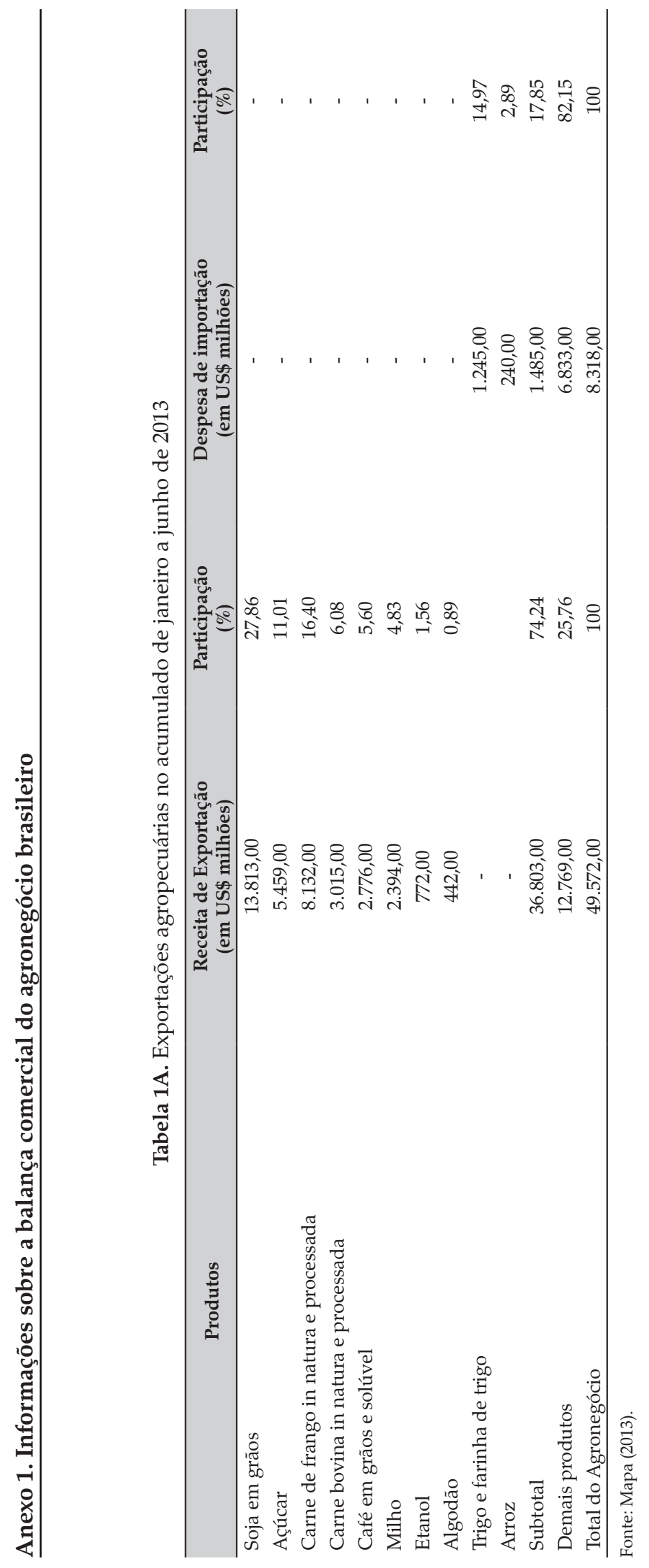

RESR, Piracicaba-SP, Vol. 53, № 02, p. 211-228, Abr/Jun 2015 - Impressa em Julho de 2015 


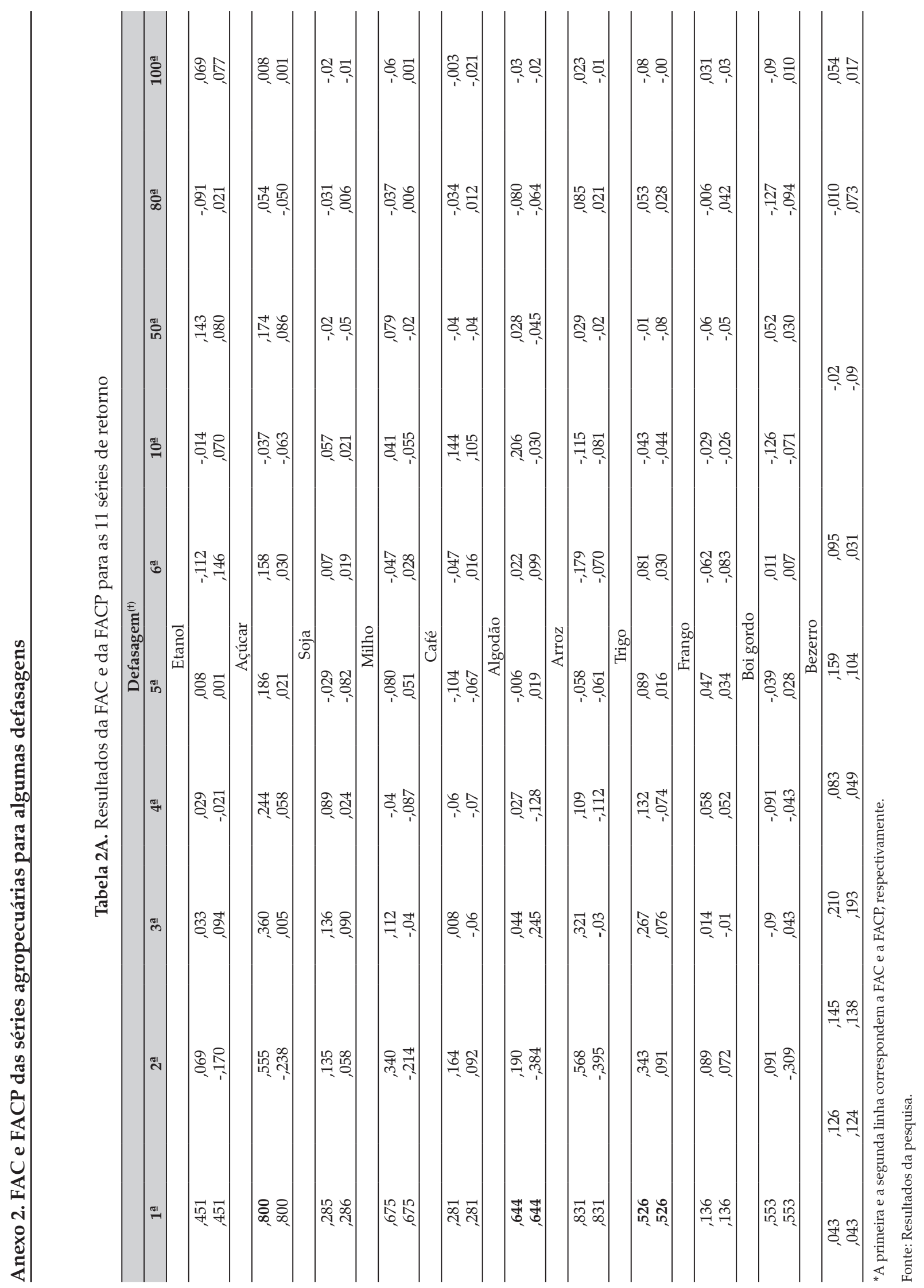


